Pacific

Journal of

Mathematics

BOUNDING THE BENDING OF A HYPERBOLIC 3-MANIFOLD

MARTIN BRIDGEMAN AND RICHARD D. CANARY 


\title{
BOUNDING THE BENDING OF A HYPERBOLIC 3-MANIFOLD
}

\author{
MARTIN BRIDGEMAN AND RICHARD D. CANARY
}

\begin{abstract}
We obtain bounds on the total bending of the boundary of the convex core of a hyperbolic 3-manifold. These bounds will depend on the geometry of the boundary of the convex hull of the limit set.
\end{abstract}

\section{Introduction}

The boundary of the convex core of a hyperbolic 3-manifold is a hyperbolic surface in its intrinsic metric. This surface is totally geodesic except along a lamination, called the bending lamination. The bending lamination inherits a transverse measure that keeps track of how much the surface is bent along the lamination. The length (or mass) of the bending lamination, regarded as a measured lamination, records the total bending of the boundary of the convex core. For example, if the boundary of the convex core is bent by an angle of $\theta$ along a single simple closed geodesic of length $L$, then the length of the bending lamination is $L \theta$.

Our main result is an upper bound on the mass of the bending lamination, which depends on a lower bound for the injectivity radius of the boundary of the convex hull of the limit set. An upper bound on the mass of the bending lamination is also implicit in the techniques developed by Bonahon and Otal [2001, Lemma 12].

If $N=\mathbb{M}^{3} / \Gamma$ is an orientable hyperbolic 3-manifold and $\Gamma$ is a nonabelian group of orientation-preserving isometries of $\mathbb{t}^{3}$, the limit set $L_{\Gamma}$ of $\Gamma$ is the smallest closed nonempty $\Gamma$-invariant subset of $\partial_{\infty} \mathbb{H}^{3}=\hat{\mathbb{C}}$. The convex core $C(N)$ of $N$ is $C H\left(L_{\Gamma}\right) / \Gamma$, where $C H\left(L_{\Gamma}\right)$ is the convex hull of $L_{\Gamma}$ in $\mathbb{H}^{3}$. Notice that $\rho_{0}$ is a lower bound for the injectivity radius of the boundary $2 \mathrm{CH}\left(L_{\Gamma}\right)$ of the convex hull of the limit set if and only if $2 \rho_{0}$ is a lower bound for the length of a compressible curve on the boundary of the convex core (i.e., a closed curve in $\partial C(N)$ that is null-homotopic in $C(N)$ but not in $\partial C(N)$ ).

Theorem 1. There exist constants $S$ and $T$ such that if $N$ is an orientable hyperbolic 3-manifold with finitely generated, nonabelian fundamental group and

Research supported in part by grants from the National Science Foundation.

MSC2000: 57M50.

Keywords: hyperbolic 3-manifold, convex core, bending lamination. 
bending lamination $\beta_{N}$ and $\rho_{0} \in(0,1]$ is a lower bound for the injectivity radius of the boundary $\partial \mathrm{CH}\left(L_{\Gamma}\right)$ of the convex hull of the limit set, then

$$
l_{\partial C(N)}\left(\beta_{N}\right) \leq|\chi(\partial C(N))|\left(S \log \frac{1}{\rho_{0}}+T\right),
$$

where $l_{\partial C(N)}\left(\beta_{N}\right)$ is the length of $\beta_{N}$ and $\chi(\partial C(N))$ is the Euler characteristic of the boundary of the convex core.

We also obtain a lower bound for the mass of the bending lamination in the case that $\partial C(N)$ has a short compressible curve. This lower bound makes clear that the dependence on the geometry of the convex hull of the limit set in our first result cannot be removed and that the form of the estimate cannot be substantially improved. Also, notice that if one passes to a degree- $d$ cover of $N$, both the length of the bending lamination and the Euler characteristic of the boundary of the convex core get multiplied by $d$, while the convex hull of the limit set is the same, so any upper bound must depend linearly on $|\chi(\partial C(N))|$.

Theorem 2. Let $N=\mathbb{H}^{3} / \Gamma$ be an orientable hyperbolic 3-manifold with finitely generated, nonabelian fundamental group. If $\partial \mathrm{CH}\left(L_{\Gamma}\right)$ contains a closed geodesic of length $\rho \leq 2 \sinh ^{-1} 1$, then

$$
l_{\partial C(N)}\left(\beta_{N}\right) \geq 4 \pi \log \frac{4 \sinh ^{-1} 1}{\rho} .
$$

If the boundary of the convex core is incompressible, Proposition 4.2 gives the following stronger result:

Theorem 3. If $N$ is an orientable hyperbolic 3-manifold with finitely generated, nonabelian fundamental group and $\partial C(N)$ is incompressible in $N$, then

$$
l_{\partial C(N)}\left(\beta_{N}\right) \leq \frac{\pi^{3}}{\sinh ^{-1} 1}|\chi(\partial C(N))| .
$$

In related work, Epstein, Marden and Markovic (see, for example, [Epstein et al. 2004, Theorem 4.2] have studied the possible bending laminations of embedded convex hyperbolic planes in $\mathbb{H}^{3}$.

Thurston [1979] (see also [Kourouniotis 1985; Johnson and Millson 1987; Epstein and Marden 1987]) studied the operation of obtaining a quasifuchsian group by bending a Fuchsian group along a simple closed geodesic, or more generally along a measured lamination. Theorem 3 may be used to quantify the observation that if this geodesic is "long," one may only bend by a "small" angle.

This paper is based on earlier work [Bridgeman 1998; Bridgeman and Canary 2003; Canary 2001], which explored the relationship between the boundary of the convex core and the conformal boundary. In particular, we make central use of a 
result (repeated here as Lemma 3.1) that ensures the existence of a lower bound, depending only on the injectivity radius of its basepoint, for the length of a geodesic arc in $\partial C H\left(L_{\Gamma}\right)$ whose intersection with the bending lamination is at least $2 \pi$. We will combine this estimate with a Crofton-like formula (Lemma 4.1) for the length of the bending lamination to prove Theorem 1 .

In Section 7, we will apply the results of [Bridgeman and Canary 2003] and [Canary 2001] to obtain analogues of Theorems 1 and 2, which depend on the geometry of the domain of discontinuity $\Omega(\Gamma)$ for the action of $\Gamma$ on $\hat{\mathbb{C}}$.

\section{Background}

Let $N=\mathbb{H}^{3} / \Gamma$ be an orientable hyperbolic 3-manifold with nonabelian fundamental group. Then $\Gamma$ acts properly discontinuously on the domain of discontinuity $\Omega(\Gamma)=\hat{\mathbb{C}}-L_{\Gamma}$. The domain of discontinuity admits a canonical conformally invariant hyperbolic metric $p(z)|d z|$, called the Poincaré metric. The quotient surface $\partial_{c} N=\Omega(\Gamma) / \Gamma$, called the conformal boundary of $N$, is then naturally a hyperbolic surface. The hyperbolic 3-manifold $N$ is said to be analytically finite if $\partial_{c} N$ has finite area in this metric. Ahlfors' Finiteness Theorem [Ahlfors 1964] asserts that $N$ is analytically finite if $\Gamma$ is finitely generated. All of our results hold for analytically finite hyperbolic 3-manifolds.

If $N$ is analytically finite then there is always a positive lower bound for the injectivity radius on $\Omega(\Gamma)$. By Lemma 8.1 of [Bridgeman and Canary 2003], a lower bound on the injectivity radius of $\Omega(\Gamma)$ implies a lower bound on the injectivity radius of $\partial \mathrm{CH}\left(L_{\Gamma}\right)$. In particular, if $N$ is analytically finite then there is a positive lower bound on the injectivity radius of $\partial C H\left(L_{\Gamma}\right)$. The boundary of the convex hull of the limit set is a hyperbolic surface in its intrinsic metric and is totally geodesic in the complement of a closed union $\beta_{\Gamma}$ of disjoint geodesics, called the bending lamination of $C H\left(L_{\Gamma}\right)$. The bending lamination $\beta_{N}$ of the convex core $C(N)$ is simply the projection of $\beta_{\Gamma}$ to $\partial C(N)$.

A measured lamination on a hyperbolic surface $S$ consists of a closed subset $\lambda$ of $S$ that is the disjoint union of simple geodesics, together with countably additive invariant (with respect to projection along $\lambda$ ) measures on arcs transverse to $\lambda$. The bending laminations $\beta_{\Gamma}$ and $\beta_{N}$ come equipped with bending measures on arcs transverse to the lamination, which record the total bending along the arc. These bending measures give $\beta_{\Gamma}$ and $\beta_{N}$ the structure of measured laminations. Real multiples of simple closed geodesics are dense in the space $M L(S)$ of all measured laminations on a finite-area hyperbolic surface $S$. Moreover, the length of a simple closed geodesic and the intersection number of two simple closed geodesics extend naturally to continuous functions on $M L(S)$ and $M L(S) \times M L(S)$ respectively. See [Thurston 1979] or [Bonahon 2001] for fuller discussions of measured lamination 
spaces and [Thurston 1979] or [Epstein and Marden 1987] for a fuller discussion of convex cores and bending laminations.

\section{Local intersection number estimates}

In [Bridgeman and Canary 2003] we obtained bounds on the intersection of a transverse geodesic arc with the bending lamination. There we defined a function

$$
F(x)=\frac{x}{2}+\sinh ^{-1}\left(\frac{\sinh (x / 2)}{\sqrt{1-\sinh ^{2}(x / 2)}}\right)
$$

and its inverse $G(x)=F^{-1}(x)$. The function $F$ is monotonically increasing and has domain $\left(0,2 \sinh ^{-1} 1\right)$. The function $G(x)$ has domain $(0, \infty)$, has asymptotic behavior $G(x) \asymp x$ as $x$ tends to 0 , and $G(x)$ approaches $2 \sinh ^{-1} 1$ as $x$ tends to $\infty$. We define $G_{\infty}=2 \sinh ^{-1} 1 \approx 1.76275$.

Lemma 3.1 [Bridgeman and Canary 2003, Lemma 4.3]. Let $N=\mathbb{M}^{3} / \Gamma$ be an analytically finite hyperbolic 3-manifold such that $L_{\Gamma}$ is not contained in a round circle. Let $\alpha:[0,1) \rightarrow \partial C H\left(L_{\Gamma}\right)$ be a geodesic path (in the intrinsic metric on $\left.\partial C H\left(L_{\Gamma}\right)\right)$ with length $l(\alpha)$. If either

(1) $l(\alpha) \leq G\left(\operatorname{inj}_{\partial C H\left(L_{\Gamma}\right)}(\alpha(0))\right)$, or

(2) $\alpha([0,1))$ is contained in a simply connected component of $\partial C H\left(L_{\Gamma}\right)$ and $l(\alpha) \leq G_{\infty}$

then

$$
i\left(\alpha, \beta_{\Gamma}\right) \leq 2 \pi
$$

A geodesic arc $\alpha$ is either transverse to $\beta_{\Gamma}$ or contained within $\beta_{\Gamma}$, in which case we define $i\left(\alpha, \beta_{\Gamma}\right)=0$.

If $\alpha:[0,1) \rightarrow \partial C(N)$ is a geodesic in the boundary of the convex core, consider its lift $\tilde{\alpha}:[0,1) \rightarrow \partial C H\left(L_{\Gamma}\right)$. If we subdivide this lift into pieces to which Lemma 3.1 applies, as in the proof of [Bridgeman and Canary 2003, Proposition 5.1], we obtain:

Corollary 3.2. Let $N$ be an analytically finite hyperbolic 3-manifold. Let $\alpha$ : $[0,1) \rightarrow \partial C(N)$ be a geodesic path with length $l(\alpha)$. If $\alpha$ is contained in an incompressible component of $\partial C(N)$, let $G=G_{\infty}$. Otherwise, let $\rho_{\alpha}$ be a lower bound on the injectivity radius of $\partial \mathrm{CH}\left(L_{\Gamma}\right)$ at every point in $\tilde{\alpha}([0,1))$ and let $G=G\left(\rho_{\alpha}\right)$. Then

$$
i\left(\alpha, \beta_{N}\right) \leq 2 \pi\left\lceil\frac{l(\alpha)}{G}\right\rceil .
$$


Here $\lceil x\rceil$, as usual, denotes the least integer greater than or equal to $x$.

We have so far avoided, for simplicity of exposition, discussing the case that the limit set is contained in a round circle. In this case, the convex core is a totally geodesic surface with geodesic boundary. It is natural to consider the boundary of the convex core to be the double of the convex core (where one considers the two sheets of the convex core to have opposite normal vectors.) With this convention, the boundary of the convex core is still a finite-area hyperbolic surface with boundary if our manifold is analytically finite. One can easily see, just as in the proof of [Bridgeman and Canary 2003, Proposition 5.1], that Corollary 3.2 remains valid in this situation.

\section{A length formula}

In order to prove Theorem 1 we first represent the length of the bending lamination as the integral of the intersection number over all geodesics of a fixed length. Our formula is similar to the Crofton formula for the area of a region in the plane. See also [Bonahon 1988, Proposition 14].

Let $S$ be a hyperbolic surface. If $v \in T^{1}(S)$ is a unit tangent vector, let $\bar{\alpha}(v)$ : $(0, \infty) \rightarrow S$ be the unit-speed geodesic ray originating at the basepoint of $v$ and in the direction of $v$. Let $\alpha^{L}(v)=\left.\bar{\alpha}\right|_{(0, L)}$ be the open geodesic segment of length $L$ emanating from the basepoint of $v$ in the direction $v$.

Lemma 4.1. Let $\beta$ be a measured lamination on a hyperbolic surface $S$ of finite area. Then

$$
l_{S}(\beta)=\frac{1}{4 L} \int_{T^{1}(S)} i\left(\alpha^{L}(v), \beta\right) d \Omega(v),
$$

where $d \Omega$ is the volume form on $T_{1}(S)$.

Proof. We define a function $F_{L}$ on the space $M L(S)$ of measured laminations by setting

$$
F_{L}(\beta)=\frac{1}{4 L} \int_{T_{1}(S)} i\left(\alpha^{L}(v), \beta\right) d \Omega(v) .
$$

As $F_{L}$ and $l_{S}$ are both continuous on $M L(S)$ and real multiples of closed geodesics are dense in $M L(S)$, it suffices to prove that $F_{L}(\beta)=l_{S}(\beta)$ for real multiples of closed geodesics. Since $F_{L}(k \beta)=k F_{L}(\beta)$ and $l_{S}(k \beta)=k l_{S}(\beta)$ for all $\beta \in M L(S)$ and all $k>0$, we may assume that $\beta$ is a single closed geodesic with unit transverse measure.

Let $C$ be the hyperbolic cylinder covering $S$ corresponding to $\beta$ and let $\tilde{\beta}$ be the lift of $\beta$ to $C$. If $v \in T^{1}(S)$, then $i\left(\alpha^{L}(v), \beta\right)$ is precisely the number of lifts of $\alpha^{L}(v)$ to $C$ that intersect $\tilde{\beta}$. Let

$$
U=\left\{v \in T^{1}(C) \mid \alpha^{L}(v) \text { intersects } \tilde{\beta}\right\} .
$$


Lifting the integral to $C$ we see that

$$
\int_{T^{1}(S)} i\left(\alpha^{L}(v), \beta\right) d \Omega(v)=\int_{U} d \Omega(v) .
$$

The metric on $C$ is given by

$$
d s^{2}=d x^{2}+\cosh ^{2} x d l^{2}
$$

where $x$ is the perpendicular distance to the core geodesic and $l$ is a length coordinate along the core geodesic; see [Buser 1992, Example 1.3.2]. The hyperbolic area element is $d A=\cosh x d x d l$.

Let

$$
N=\{c \in C \mid 0<d(\widetilde{\beta}, c)<L\} .
$$

If $v \in U$, the basepoint $p$ of $v$ is in $N$. If $p \in N$, let $U_{p}$ denote the cone of tangent vectors in $U \cap T_{p}^{1}(C)$. Let $w_{p}$ denote the unit vector tangent to the geodesic ray through $p$ perpendicular to $\widetilde{\beta}$. Then $U_{p}$ consists of all vectors in $T_{p}^{1}(C)$ making an angle of at most $\theta(p)$ with $w_{p}$, where

$$
\theta(p)=\cos ^{-1} \frac{\tanh x}{\tanh L} .
$$

Therefore,

$$
\int_{U} d \Omega(v)=\int_{N} 2 \cos ^{-1} \frac{\tanh x}{\tanh L} d A .
$$

Integrating over the core of the annulus we obtain

$$
\begin{aligned}
\int_{N} 2 \cos ^{-1} \frac{\tanh x}{\tanh L} d A & =2 l_{S}(\beta) \int_{-L}^{L} \cosh x \cos ^{-1} \frac{\tanh x}{\tanh L} d x \\
& =4 l_{S}(\beta) \int_{0}^{L} \cosh x \cos ^{-1} \frac{\tanh x}{\tanh L} d x
\end{aligned}
$$

Therefore,

$$
F_{L}(\beta)=\frac{l_{S}(\beta)}{L} \int_{0}^{L} \cosh x \cos ^{-1} \frac{\tanh x}{\tanh L} d x .
$$

Substituting $u=\frac{\tanh x}{\tanh L}$ we obtain

$$
F_{L}(\beta)=\frac{l_{S}(\beta) \tanh L}{L} \int_{0}^{1} \frac{\cos ^{-1} u}{\left(1-u^{2} \tanh ^{2} L\right)^{3 / 2}} d u .
$$

We may then integrate by parts and evaluate the result to check that $F_{L}(\beta)$ has the claimed form. 
We now prove a version of Theorem 1 that is a direct application of Corollary 3.2 and Lemma 4.1. Recall that $G(x) \asymp x$ as $x$ tends to 0 . If $\rho_{0} \geq 1$, this estimate is better than the one provided by Theorem 1 , but it is much weaker as $\rho_{0}$ approaches 0 , since the upper bound provided by Proposition 4.2 is $O\left(|\chi(\partial C(N))| / \rho_{0}\right)$, while the estimate provided by Theorem 1 is $O\left(|\chi(\partial C(N))| \log \left(\rho_{0}^{-1}\right)\right)$. Notice that Theorem 3 is case (2) of Proposition 4.2.

Proposition 4.2. Let $N=\mathbb{H}^{3} / \Gamma$ be an analytically finite hyperbolic 3-manifold with bending lamination $\beta_{N}$.

(1) If $\rho_{0}>0$ is a lower bound for the injectivity radius of $\partial \mathrm{CH}\left(L_{\Gamma}\right)$, then

$$
l_{\partial C(N)}\left(\beta_{N}\right) \leq \frac{2 \pi^{3}}{G\left(\rho_{0}\right)}|\chi(\partial C(N))| .
$$

(2) If $\partial C(N)$ is incompressible in $N$, then

$$
l_{\partial C(N)}\left(\beta_{N}\right) \leq \frac{\pi^{3}}{\sinh ^{-1} 1}|\chi(\partial C(N))| .
$$

Proof. If $\partial C(N)$ is incompressible, we let $G=G_{\infty}=2 \sinh ^{-1} 1$. If not, we let $G=G\left(\rho_{0}\right)$. Corollary 3.2 implies that, for all $v \in T^{1}(\partial C(N))$,

$$
i\left(\alpha^{L}(v), \beta_{N}\right) \leq 2 \pi\left\lceil\frac{L}{G}\right\rceil \leq 2 \pi\left(\frac{L}{G}+1\right) .
$$

Therefore, by Lemma 4.1,

$$
l_{\partial C(N)}\left(\beta_{N}\right) \leq \frac{\pi}{2 L} \int_{T^{1}(\partial C(N))}\left(\frac{L}{G}+1\right) d \Omega \leq \operatorname{vol} T^{1}(\partial C(N))\left(\frac{\pi}{2 G}+\frac{\pi}{2 L}\right) .
$$

The volume of the unit tangent bundle $T^{1}(\partial C(N))$ is $4 \pi^{2}|\chi(\partial C(N))|$. Thus, by letting $L$ tend to infinity, we see that

$$
l_{\partial C(N)}\left(\beta_{N}\right) \leq 4 \pi^{2}|\chi(\partial C(N))|\left(\frac{\pi}{2 G}\right)=\frac{2 \pi^{3}}{G}|\chi(\partial C(N))| .
$$

\section{Proof of Theorem 1}

To obtain the sharper bound on the length of the bending lamination given by Theorem 1, we must decompose $\partial C(N)$ using the Collar Lemma. We will use the following explicit version of the Collar Lemma, which combines [Buser 1992, Theorem 4.4.6] and [Yamada 1982, Lemma 7] (which guarantees that curves of length at most $2 \sinh ^{-1} 1$ are simple). 
Collar Lemma. Let $S$ be a finite-area hyperbolic surface of genus $g$ with $n$ punctures. Let $\left\{v_{1}, \ldots, v_{k}\right\}$ be the collection of all primitive closed geodesics on $S$ of length at most $2 \sinh ^{-1} 1$. Then:

(1) $k \leq 3 g-3+n$.

(2) $\left\{v_{1}, \ldots, v_{k}\right\}$ is a disjoint collection of simple closed geodesics.

(3) There exists a disjoint collection $\left\{B_{1}, \ldots, B_{k}\right\}$ of metric collar neighborhoods of $\left\{v_{1}, \ldots, v_{k}\right\}$ such that each $B_{i}$ is isometric to the quotient of

$$
\left[-w\left(v_{i}\right), w\left(v_{i}\right)\right] \times\left[0, l_{S}\left(v_{i}\right)\right]
$$

by the identification map $(t, 0) \mapsto\left(t, l_{S}\left(v_{i}\right)\right)$, where $l_{S}\left(v_{i}\right)$ is the length of $v_{i}$,

$$
w\left(v_{i}\right)=\sinh ^{-1} \frac{1}{\sinh \left(\frac{1}{2} l_{S}\left(v_{i}\right)\right)},
$$

and the product has the metric

$$
d s^{2}=d x^{2}+\cosh ^{2} x d l^{2} .
$$

(4) If $x \in B_{i}$, then $\sinh \operatorname{inj}_{S}(x)=\sinh \left(\frac{1}{2} l_{S}\left(v_{i}\right)\right) \cosh d\left(x, v_{i}\right)$.

(5) If there is a curve through $x \in S$ homotopic to $v_{i}$ of length at most $2 \sinh ^{-1} 1$, then $x \in B_{i}$.

We now restate Theorem 1 for analytically finite hyperbolic 3-manifolds.

Theorem 1. There exist constants $S$ and $T$ such that if $N=\mathbb{H}^{3} / \Gamma$ is an analytically finite hyperbolic 3-manifold with bending lamination $\beta_{N}$ and $\rho_{0} \in(0,1]$ is a lower bound for the injectivity radius of the boundary $\partial \mathrm{CH}\left(L_{\Gamma}\right)$ of the convex hull of the limit set, then

$$
l_{\partial C(N)}\left(\beta_{N}\right) \leq|\chi(\partial C(N))|\left(S \log \frac{1}{\rho_{0}}+T\right),
$$

where $l_{\partial C(N)}\left(\beta_{N}\right)$ is the length of $\beta_{N}$ and $\chi(\partial C(N))$ is the Euler characteristic of the boundary of the convex core.

Proof. As the proof is rather technical, we begin with a brief outline. We first decompose $\partial C(N)$ into the set $X$ of collars of short compressible geodesics and its complement $Y$. We choose $\epsilon=\sinh ^{-1} 1$ and $L=G(\epsilon)$. By Lemma 4.1

$$
\begin{aligned}
l_{\partial C(N)}\left(\beta_{N}\right) & =\frac{1}{4 L} \int_{T^{1}(S)} i\left(\alpha^{L}(v), \beta_{N}\right) d \Omega(v) \\
& =\frac{1}{4 L}\left(\int_{T^{1}(X)} i\left(\alpha^{L}(v), \beta_{N}\right) d \Omega(v)+\int_{T^{1}(Y)} i\left(\alpha^{L}(v), \beta_{N}\right) d \Omega(v)\right) .
\end{aligned}
$$


Lemma 3.1 implies that $i\left(\alpha^{L}(v), \beta_{N}\right) \leq 2 \pi$ for $v \in T^{1}(Y)$, so, just as in the proof of Proposition 4.2,

$$
\int_{T^{1}(Y)} i\left(\alpha^{G(\epsilon)}(v), \beta_{N}\right) d \Omega \leq 2 \pi \operatorname{vol} T^{1}(Y) .
$$

To handle the integral over $T^{1}(X)$, we use Corollary 3.2, which implies that

$$
i\left(\alpha^{L}(v), \beta_{N}\right) \leq 2 \pi\left\lceil\frac{L}{G(r(v))}\right\rceil,
$$

where $r(v)$ is a lower bound on the injectivity radius of $\partial C(N)$ at any point on $\alpha^{L}(v)$. If $B$ is a component of $X$ with core geodesic $v$ and $v \in T^{1}(B)$, we observe that

$$
r(v) \geq \sinh ^{-1}\left(\frac{1}{e^{G(\epsilon)}} \sinh \frac{l_{S}(v)}{2} \cosh d(v)\right)
$$

where $d(v)$ is the distance from the basepoint of $v$ to $v$. Combining the resulting bounds and integrating, we obtain an upper bound on the integral of $i\left(\alpha^{L}(v), \beta_{N}\right)$ over $T^{1}(B)$ in terms of the length of $v$. Summing the resulting bounds over $T^{1}(Y)$ and all components of $T^{1}(X)$ gives our result.

Let $\left\{v_{1}, \ldots, v_{k}\right\}$ be the primitive closed geodesics of length at most $2 \sinh ^{-1} 1$ on $\partial C(N)$. Let $\left\{B_{1}, \ldots, B_{k}\right\}$ be the collar neighborhoods of $\left\{v_{1}, \ldots, v_{k}\right\}$ provided by the Collar Lemma.

Let $\pi: \partial C H\left(L_{\Gamma}\right) \rightarrow \partial C(N)$ be the covering map from the boundary of the convex hull to the boundary of the convex core. Set $\epsilon=\sinh ^{-1} 1$ and

$$
\widetilde{V}=\left\{x \in \partial C H\left(L_{\Gamma}\right) \mid \operatorname{inj}_{\partial C H\left(L_{\Gamma}\right)}(x) \leq \epsilon\right\} .
$$

If $x \in \widetilde{V}$, then $x$ lies on a homotopically nontrivial curve $n_{x}$ of length at most $2 \epsilon$. Since there is a lower bound on the injectivity radius of $\partial C H\left(L_{\Gamma}\right), n_{x}$ is homotopic to a closed geodesic $\tilde{v}_{x}$ of length at most $2 \epsilon$. Then $\tilde{v}_{x}$ projects to (a multiple of) one of the curves $\left\{v_{1}, \ldots, v_{k}\right\}$, so $\pi(x)$ lies in some collar neighborhood $B_{i}$ and $x$ lies in a lift of $B_{i}$ to $\partial C H\left(L_{\Gamma}\right)$. Let $X$ denote the union of all collar neighborhoods $B_{i}$ containing some component of $\pi(\tilde{V})$. Let $Y=\partial C(N)-X$. We may renumber $\left\{B_{1}, \ldots, B_{k}\right\}$ so that $X=\bigcup_{i=1}^{m} B_{i}$ for some $m \leq k$. Notice that $\operatorname{inj}_{\partial C H\left(L_{\Gamma}\right)}(y)>\epsilon$ for $y \in \pi^{-1}(Y)$.

We choose $L=G(\epsilon)$ in the formula for $l_{\partial C(N)}\left(\beta_{N}\right)$ in Lemma 4.1. We split the integral into two integrals using the decomposition, so that

$$
l_{\partial C(N)}\left(\beta_{N}\right)=\frac{1}{4 G(\epsilon)}\left(\int_{T^{1}(X)} i\left(\alpha^{G(\epsilon)}(v), \beta_{N}\right) d \Omega+\int_{T^{1}(Y)} i\left(\alpha^{G(\epsilon)}(v), \beta_{N}\right) d \Omega\right) .
$$

We first estimate the portion of the integral with domain $T^{1}(Y)$. If $v$ has basepoint in $Y$ and $\tilde{\alpha}^{G(\epsilon)}(v)$ is a lift of $\alpha^{G(\epsilon)}(v)$ to $\partial C H\left(L_{\Gamma}\right)$, then $\tilde{\alpha}^{G(\epsilon)}(v)$ originates 
at a point $\tilde{y}$ such that $\operatorname{inj}_{\partial C H\left(L_{\Gamma}\right)}(\tilde{y})>\epsilon$ and has length $G(\epsilon)<G\left(\operatorname{inj}_{\partial C H\left(L_{\Gamma}\right)}(\tilde{y})\right)$. Therefore, Lemma 3.1 implies that $i\left(\widetilde{\alpha}^{G(\epsilon)}(v), \beta_{\Gamma}\right) \leq 2 \pi$ and hence that

$$
i\left(\alpha^{G(\epsilon)}(v), \beta_{N}\right) \leq 2 \pi \text {. }
$$

Therefore

$$
\int_{T^{1}(Y)} i\left(\alpha^{G(\epsilon)}(v), \beta_{N}\right) d \Omega \leq \int_{T^{1}(Y)} 2 \pi d \Omega \leq 2 \pi \operatorname{vol} T^{1}(Y) .
$$

We now estimate the portion of the integral with domain $T^{1}(X)$. If $X$ is empty, we are done. Otherwise, let $B_{i}$ be a component of $X$. Let $v \in T_{1}\left(B_{i}\right)$ and $d_{i}(v)$ be the distance from $v_{i}$ to the basepoint $b_{v}$ of $v$.

We now derive a lower bound for the injectivity radius along the geodesic $\alpha^{G(\epsilon)}(v)$ as a function of $d_{i}(v)$. One can readily check that if $S$ is a hyperbolic surface, $w, z \in S$ and $\delta=d_{S}(z, w)$, then $\sinh \operatorname{inj}_{S}(w) \geq e^{-\delta} \sinh _{\operatorname{inj}_{S}}(z)$. (This follows, for example, from [Beardon 1983, Theorem 7.35.1].) Since, by the Collar Lemma,

$$
\sinh \operatorname{inj}_{S}\left(b_{v}\right)=\sinh \frac{l_{S}\left(v_{i}\right)}{2} \cosh d_{i}(v),
$$

we see that if $x$ is any point on $\alpha^{G(\epsilon)}(v)$, then

$$
\sinh \operatorname{inj}_{\partial C(N)}(x) \geq \frac{1}{e^{G(\epsilon)}} \sinh \frac{l_{S}\left(v_{i}\right)}{2} \cosh d_{i}(v) .
$$

We define $R_{i}:\left[0, w\left(v_{i}\right)\right] \rightarrow \mathbb{R}$ by

$$
R_{i}(t)=\sinh ^{-1}\left(\frac{1}{e^{G(\epsilon)}} \sinh \frac{l_{S}\left(v_{i}\right)}{2} \cosh t\right) .
$$

The injectivity radius at any point of $\alpha^{G(\epsilon)}(v)$ is bounded from below by $R_{i}\left(d_{i}(v)\right)$, so if $\tilde{\alpha}^{G(\epsilon)}(v)$ is a lift of $\alpha^{G(\epsilon)}(v)$ to $\partial C H\left(L_{\Gamma}\right)$, the injectivity radius of $\partial C H\left(L_{\Gamma}\right)$ at every point of $\tilde{\alpha}^{G(\epsilon)}(v)$ is also bounded from below by $R_{i}\left(d_{i}(v)\right)$. Thus, by Corollary 3.2 ,

$$
i\left(\alpha^{G(\epsilon)}(v), \beta_{N}\right) \leq 2 \pi\left\lceil\frac{G(\epsilon)}{G\left(R_{i}\left(d_{i}(v)\right)\right)}\right\rceil .
$$

So

$$
\begin{aligned}
& \int_{T^{1}\left(B_{i}\right)} i\left(\alpha^{G(\epsilon)}(v), \beta_{N}\right) d \Omega \leq \int_{T^{1}\left(B_{i}\right)} 2 \pi\left\lceil\frac{G(\epsilon)}{G\left(R_{i}\left(d_{i}(v)\right)\right)}\right\rceil d \Omega \\
& \leq 2 \pi G(\epsilon) \int_{T^{1}\left(B_{i}\right)} \frac{1}{G\left(R_{i}\left(d_{i}(v)\right)\right)} d \Omega+2 \pi \operatorname{vol} T^{1}\left(B_{i}\right) .
\end{aligned}
$$

Since the integral depends only on $d_{i}(v)$,

$$
\int_{T^{1}\left(B_{i}\right)} \frac{1}{G\left(R_{i}\left(d_{i}(v)\right)\right)} d \Omega \leq 2 \pi \int_{0}^{l_{S}\left(v_{i}\right)} \int_{-\omega\left(v_{i}\right)}^{\omega\left(v_{i}\right)} \frac{1}{G\left(R_{i}(|x|)\right)} \cosh x d x d l,
$$


where $x$ and $l$ are the coordinates on $B_{i}$ provided by the Collar Lemma.

As $R_{i}(|x|)<\epsilon$ on $B_{i}$, we need only consider $G$ on the domain $[0, \epsilon]$. Since $t / G(t)$ tends to 1 as $t$ tends to 0 and is continuous on $(0, \epsilon]$, there exists a constant $K_{1}>0$ such that $t / G(t) \leq K_{1}$ for all $t \in(0, \epsilon]$. Therefore

$$
\int_{T^{1}\left(B_{i}\right)} \frac{1}{G\left(R_{i}\left(d_{i}(v)\right)\right)} d \Omega \leq 2 \pi \int_{0}^{l_{S}\left(v_{i}\right)} \int_{-\omega\left(v_{i}\right)}^{\omega\left(v_{i}\right)} \frac{K_{1} \cosh x}{R_{i}(|x|)} d x d l .
$$

Integrating over the core curve and making use of the symmetry about the core geodesic, we see that

$$
\int_{T^{1}\left(B_{i}\right)} \frac{1}{G\left(R_{i}\left(d_{i}(v)\right)\right)} d \Omega \leq 4 \pi K_{1} l_{S}\left(v_{i}\right) \int_{0}^{w\left(v_{i}\right)} \frac{\cosh x}{R_{i}(x)} d x .
$$

Since $\sinh x / x$ is increasing on $(0, \infty), \sinh x / x \leq K_{2}=\sinh \epsilon / \epsilon$ for all $x \in$ $(0, \epsilon]$. Thus, for all $x \in\left(0, w\left(v_{i}\right)\right)$,

$$
\frac{1}{R_{i}(x)} \leq \frac{K_{2}}{\sinh R_{i}(x)}
$$

Therefore,

$$
\int_{0}^{w\left(v_{i}\right)} \frac{\cosh x}{R_{i}(x)} d x \leq \int_{0}^{w\left(v_{i}\right)} \frac{K_{2} e^{G(\epsilon)}}{\sinh \left(l\left(v_{i}\right) / 2\right)} d x \leq \frac{w\left(v_{i}\right) K_{2} e^{G(\epsilon)}}{\sinh \left(l\left(v_{i}\right) / 2\right)} .
$$

Combining inequalities (3) and (4) we see that

$$
\int_{T^{1}\left(B_{i}\right)} \frac{1}{G\left(R_{i}\left(d_{i}(v)\right)\right)} d \Omega \leq \frac{4 \pi K_{1} l\left(v_{i}\right) w\left(v_{i}\right) K_{2} e^{G(\epsilon)}}{\sinh \left(l\left(v_{i}\right) / 2\right)} .
$$

Since $\sinh x \geq x$, we get

$$
\int_{T^{1}\left(B_{i}\right)} \frac{1}{G\left(R_{i}\left(d_{i}(v)\right)\right)} d \Omega \leq 8 \pi K_{1} K_{2} e^{G(\epsilon)} w\left(v_{i}\right) .
$$

Applying the equality $\sinh ^{-1} x=\log \left(x+\sqrt{x^{2}+1}\right)$, we see that

$$
w\left(v_{i}\right)=\sinh ^{-1} \frac{1}{\sinh \left(l\left(v_{i}\right) / 2\right)}=\log \frac{1+\cosh \left(l\left(v_{i}\right) / 2\right)}{\sinh \left(l\left(v_{i}\right) / 2\right)} .
$$

Thus

$$
\begin{aligned}
w\left(v_{i}\right) & \leq \log \left(1+\cosh \frac{l\left(v_{i}\right)}{2}\right)+\log \frac{1}{\sinh \left(l\left(v_{i}\right) / 2\right)} \\
& \leq \log (1+\cosh \epsilon)+\log \frac{2}{l\left(v_{i}\right)} .
\end{aligned}
$$


This yields

$$
\int_{T^{1}\left(B_{i}\right)} \frac{1}{G\left(R_{i}\left(d_{i}(v)\right)\right)} d \Omega \leq S_{0} \log \frac{2}{l\left(v_{i}\right)}+T_{0},
$$

where $S_{0}=8 \pi K_{1} K_{2} e^{G(\epsilon)}$ and $T_{0}=S_{0} \log (1+\cosh \epsilon)$.

Since $X=\bigcup_{i=1}^{m} B_{i}$, we can combine inequalities (2) and (5) to obtain

$$
\begin{aligned}
\int_{T^{1}(X)} i\left(\alpha^{G(\epsilon)}(v), \beta_{N}\right) d \Omega & =\sum_{i=1}^{m} \int_{T^{1}\left(B_{i}\right)} i\left(\alpha^{G(\epsilon)}(v), \beta_{N}\right) d \Omega \\
& \leq \sum_{i=1}^{m} 2 \pi G(\epsilon)\left(S_{0} \log \frac{2}{l\left(v_{i}\right)}+T_{0}\right)+2 \pi \operatorname{vol} T^{1}\left(B_{i}\right)
\end{aligned}
$$

Since $m$ is bounded above by the number of disjoint geodesics in $\partial C(N)$,

$$
m \leq \frac{3}{2}|\chi(\partial C(N))| .
$$

Moreover, as $\rho_{0}$ is a lower bound for the injectivity radius of $\partial C H\left(L_{\Gamma}\right)$, we have $\rho_{0} \leq l\left(v_{i}\right) / 2$ for all $i$. Therefore,

$$
\begin{aligned}
\int_{T^{1}(X)} i\left(\alpha^{G(\epsilon)}(v),\right. & \left.\beta_{N}\right) d \Omega \\
& \leq 3 \pi G(\epsilon)|\chi(\partial C(N))|\left(S_{0} \log \frac{1}{\rho_{0}}+T_{0}\right)+2 \pi \operatorname{vol} T^{1}(X)
\end{aligned}
$$

Combining estimates (1) and (6) for the integral over $T^{1}(X)$ and $T^{1}(Y)$, we get

$$
\begin{aligned}
\int_{T^{1}(\partial C(N))} & i\left(\alpha^{G(\epsilon)}(v), \beta_{N}\right) d \Omega \\
= & \int_{T^{1}(X)} i\left(\alpha^{G(\epsilon)}(v), \beta_{N}\right) d \Omega+\int_{T^{1}(Y)} i\left(\alpha^{G(\epsilon)}(v), \beta_{N}\right) d \Omega \\
\leq & 3 \pi G(\epsilon)|\chi(\partial C(N))|\left(S_{0} \log \frac{1}{\rho_{0}}+T_{0}\right)+2 \pi \operatorname{vol} T^{1}(X)+2 \pi \operatorname{vol} T^{1}(Y) \\
\leq & 3 \pi G(\epsilon)|\chi(\partial C(N))|\left(S_{0} \log \frac{1}{\rho_{0}}+T_{0}\right)+2 \pi \operatorname{vol} T^{1}(\partial C(N)) .
\end{aligned}
$$

Recalling that

$$
l_{\partial C(N)}\left(\beta_{N}\right)=\frac{1}{4 G(\epsilon)} \int_{T^{1}(\partial C(N))} i\left(\alpha^{G(\epsilon)}(v), \beta_{N}\right) d \Omega
$$

and that vol $T^{1}(\partial C(N))=4 \pi^{2}|\chi(\partial C(N))|$, we see that this implies that

$$
l_{\partial C(N)}\left(\beta_{N}\right) \leq|\chi(\partial C(N))|\left(S \log \frac{1}{\rho_{0}}+T\right),
$$


where

$$
S=\frac{3 \pi S_{0}}{4} \quad \text { and } \quad T=\frac{3 \pi T_{0}}{4}+\frac{2 \pi^{3}}{G(\epsilon)} .
$$

Remark. One can evaluate the constants used in the proof to check that $\epsilon=$ $\sinh ^{-1} 1 \approx 0.8814, G(\epsilon)=F^{-1}(\epsilon) \approx 0.8387, K_{1}=\epsilon / G(\epsilon) \approx 1.0509$ (since $t / G(t)$ is increasing), and $K_{2}=\sinh \epsilon / \epsilon \approx 1.1346$. Therefore, $S \leq 164$ and $T \leq 218$.

\section{A lower bound on the length of the bending lamination}

If the boundary of the convex core contains a short compressible curve we obtain a lower bound on the length of the bending lamination, having the same asymptotic form as the upper bound obtained in Theorem 1. Notice that if $N$ is Fuchsian, the bending lamination has length zero, so no general lower bound is possible.

Theorem 2. Let $N=\mathbb{M}^{3} / \Gamma$ be an analytically finite hyperbolic 3-manifold. If $\partial C H\left(L_{\Gamma}\right)$ contains a closed geodesic of length $\rho \leq 2 \sinh ^{-1} 1$, then

$$
l_{\partial C(N)}\left(\beta_{N}\right) \geq 4 \pi \log \frac{4 \sinh ^{-1} 1}{\rho} .
$$

Proof. Let $\tilde{\alpha}$ be the closed geodesic of length $\rho$ on $\partial C H\left(L_{\Gamma}\right)$ and let $\epsilon=\sinh ^{-1} 1$. Let $\alpha$ be the projection of $\tilde{\alpha}$ to $\partial C(N)$. It follows from the Collar Lemma that $\alpha$ is a multiple of a simple closed geodesic $\nu$. Let $B$ be the collar of $v$ provided by the Collar Lemma. The collar $B$ has width $w \geq \sinh ^{-1}(1 / \sinh (\rho / 2))$. Since $\sinh ^{-1} x=\log \left(x+\sqrt{x^{2}+1}\right)$,

$$
w \geq \log \frac{1+\cosh (\rho / 2)}{\sinh (\rho / 2)} \geq \log \frac{2}{\sinh (\rho / 2)} .
$$

Since $\sinh x / x$ is an increasing function on $(0, \infty)$,

$$
\sinh \frac{\rho}{2} \leq \frac{\sinh \epsilon}{\epsilon} \frac{\rho}{2}=\frac{\rho}{2 \epsilon},
$$

so

$$
w \geq \log \frac{4 \epsilon}{\rho} .
$$

Any leaf of $\beta_{N} \cap B$ that intersects $\alpha$ intersects it exactly once and runs from one boundary component of $B$ to the other and has length at least $2 w$. By [Lecuire 2002, Proposition 4] we have $i\left(\alpha, \beta_{N}\right)>2 \pi$ (see also [Bonahon and Otal 2001, Proposition 7] for the case when $\beta_{N}$ is finite-leaved). Thus, the total (measured) length of $\beta_{N} \cap B$ is at least $2 \pi(2 w)=4 \pi w$. Therefore

$$
l_{\partial C(N)}\left(\beta_{N}\right) \geq 4 \pi \log \frac{4 \epsilon}{\rho},
$$

as claimed. 


\section{Bounds depending on the geometry of $\Omega(\Gamma)$}

We observed in [Bridgeman and Canary 2003] that a lower bound on the injectivity radius of the boundary of the convex hull implies a lower bound on the injectivity radius of the domain of discontinuity, while in [Canary 2001] we saw that a short geodesic in the domain of discontinuity implies the existence of an even shorter geodesic in the boundary of the convex hull. Therefore, we can give versions of Theorems 1 and 2 where the constants depend on the geometry of the domain of discontinuity.

If $N=\mathbb{H}^{3} / \Gamma$ is an analytically finite hyperbolic 3-manifold, then [Bridgeman and Canary 2003, Lemma 8.1] implies that

$$
\frac{1}{2} e^{-m} e^{-\pi^{2} /\left(2 r_{0}\right)}
$$

is a lower bound for the injectivity radius of $\partial C H\left(L_{\Gamma}\right)$, where $m=\cosh ^{-1} e^{2}$ and $r_{0}$ is a lower bound for the injectivity radius of the domain of discontinuity $\Omega(\Gamma)$ of $\Gamma$. Therefore, we obtain the following version of Theorem 1, where $S^{\prime}=\frac{1}{2} \pi^{2} S$ and $T^{\prime}=S \log 2+S m+T$.

Theorem 1'. There exist constants $S^{\prime}$ and $T^{\prime}$ such that if $N$ is an analytically finite hyperbolic 3-manifold with bending lamination $\beta_{N}$ and $r_{0}$ is a lower bound for the injectivity radius of the domain of discontinuity $\Omega(\Gamma)$, then

$$
l_{\partial C(N)}\left(\beta_{N}\right) \leq|\chi(\partial C(N))|\left(\frac{S^{\prime}}{r_{0}}+T^{\prime}\right),
$$

where $l_{\partial C(N)}\left(\beta_{N}\right)$ is the length of $\beta_{N}$ and $\chi(\partial C(N))$ is the Euler characteristic of the boundary of the convex core.

Theorem 5.1 of [Canary 2001] implies that if $\Omega(\Gamma)$ contains a closed geodesic of length $r \leq 1$, then $\partial C H\left(L_{\Gamma}\right)$ contains a closed geodesic of length at most

$$
\frac{4 \pi e^{0.502 \pi}}{e^{\pi^{2} /(\sqrt{e} r)}} \leq .153 r .
$$

Thus, we obtain the following version of Theorem 2 , where $P=4 \pi^{3} / \sqrt{e}$ and $Q=4 \pi \log \left(4 \pi e^{0.502 \pi} / \sinh ^{-1} 1\right)$.

Theorem 2'. There exist positive constants $P$ and $Q$ such that if $N=\mathbb{H}^{3} / \Gamma$ is an analytically finite hyperbolic 3-manifold, $\Omega(\Gamma)$ contains a closed geodesic of length $r \leq 1$, then

$$
l_{\partial C(N)}\left(\beta_{N}\right) \geq \frac{P}{r}-Q
$$




\section{References}

[Ahlfors 1964] L. V. Ahlfors, "Finitely generated Kleinian groups", Amer. J. Math. 86 (1964), 413429. MR 29 \#4890 Zbl 0133.04201

[Beardon 1983] A. F. Beardon, The geometry of discrete groups, Graduate Texts in Mathematics 91, Springer, New York, 1983. MR 85d:22026 Zbl 0528.30001

[Bonahon 1988] F. Bonahon, "The geometry of Teichmüller space via geodesic currents", Invent. Math. 92:1 (1988), 139-162. MR 90a:32025 Zbl 0653.32022

[Bonahon 2001] F. Bonahon, "Geodesic laminations on surfaces", pp. 1-37 in Laminations and foliations in dynamics, geometry and topology (Stony Brook, NY, 1998), edited by M. Lyubich et al., Contemp. Math. 269, Amer. Math. Soc., Providence, RI, 2001. MR 2001m:57023 Zbl 0996.53029

[Bonahon and Otal 2001] F. Bonahon and J.-P. Otal, "Laminations mesurées de plissage des variétés hyperboliques de dimension 3", preprint, Université d'Orléans (MAPMO), 2001, Available at http:// www.univ-orleans.fr/SCIENCES/MAPMO/publications/mapmo2001/mapmo0115.pdf.

[Bridgeman 1998] M. Bridgeman, "Average bending of convex pleated planes in hyperbolic threespace”, Invent. Math. 132:2 (1998), 381-391. MR 99f:57015 Zbl 0912.30028

[Bridgeman and Canary 2003] M. Bridgeman and R. D. Canary, "From the boundary of the convex core to the conformal boundary", Geom. Dedicata 96 (2003), 211-240. MR 2003j:57026 Zbl 01901496

[Buser 1992] P. Buser, Geometry and spectra of compact Riemann surfaces, Progress in Mathematics 106, Birkhäuser, Boston, 1992. MR 93g:58149 Zbl 0770.53001

[Canary 2001] R. D. Canary, "The conformal boundary and the boundary of the convex core", Duke Math. J. 106:1 (2001), 193-207. MR 2001m:57024 Zbl 1012.57021

[Epstein and Marden 1987] D. B. A. Epstein and A. Marden, "Convex hulls in hyperbolic space, a theorem of Sullivan, and measured pleated surfaces", pp. 113-253 in Analytical and geometric aspects of hyperbolic space (Coventry/Durham, 1984), edited by D. B. A. Epstein, London Math. Soc. Lecture Note Ser. 111, Cambridge Univ. Press, Cambridge, 1987. MR 89c:52014 Zbl 0612.57010

[Epstein et al. 2004] D. B. A. Epstein, A. Marden, and V. Markovic, "Quasiconformal homeomorphisms and the convex hull boundary”, Ann. of Math. (2) 159:1 (2004), 305-336. MR 2052356

[Johnson and Millson 1987] D. Johnson and J. J. Millson, "Deformation spaces associated to compact hyperbolic manifolds", pp. 48-106 in Discrete groups in geometry and analysis (New Haven, 1984), edited by R. Howe, Progr. Math. 67, Birkhäuser, Boston, 1987. MR 88j:22010 Zbl 0664. 53023

[Kourouniotis 1985] C. Kourouniotis, "Deformations of hyperbolic structures", Math. Proc. Cambridge Philos. Soc. 98:2 (1985), 247-261. MR 87g:32022 Zbl 0577.53041

[Lecuire 2002] C. Lecuire, "Plissage des variétés hyperboliques de dimension 3", preprint 0301, Université de Lyon, 2002.

[Thurston 1979] W. P. Thurston, "The geometry and topology of 3-manifolds", lecture notes, Princeton University, 1979, Available at http://www.msri.org/publications/books/gt3m/.

[Yamada 1982] A. Yamada, "On Marden's universal constant of Fuchsian groups, II", J. Analyse Math. 41 (1982), 234-248. MR 84h:30073 Zbl 0513.30037

Received January 3, 2003. Revised June 7, 2003. 
MARTIN BRIDGEMAN

DEPARTMENT OF MATHEMATICS

Boston COLLEGE

Chestnut Hill, MA 02167

bridgem@bc.edu

RICHARD D. CANARY

DEPARTMENT OF MATHEMATICS

UNIVERSITY OF MICHIGAN

ANN ARBOR, MI 48109-1109

canary@umich.edu 